\title{
A simple synthesis of stable phosphorus ylides containing cyano groups, from the reaction between triphenylphosphine and acetylenic esters in the presence of $\mathrm{CH}$ - acid compounds
}

\author{
Malek Taher Maghsoodlou,* Hamid Reza Shaterian, Ghasem Marandi, Fahimeh Shahraki \\ Poor, and Jaber Salehzadeh \\ Department of Chemistry, The University of Sistan \& Balouchestan, P. O. Box 98135-674, \\ Zahedan, Iran \\ E-mail: mt_maghsoodlou@yahoo.com
}

\begin{abstract}
Stable crystalline phosphorus ylides were obtained in excellent yields from the 1:1:1 addition reaction between triphenylphosphine and dialkyl acetylenedicarboxylates, in the presence of strong CH- acids such as malononitrile or ethyl cyanoacetate. These stable ylides exist in solution as a mixture of two geometrical isomers as a result of restricted rotation around the carbon-carbon partial double bond resulting from conjugation of the ylide moiety with the adjacent carbonyl group.
\end{abstract}

Keywords: Acetylenic ester, $\mathrm{CH}$ - acids, stable phosphorus ylides, triphenylphosphine, geometrical isomer

\section{Introduction}

The synthesis of phosphorus ylides is important in organic chemistry because of their applications in the synthesis of organic products, ${ }^{1}$ especially the synthesis of naturally-occurring products with potentially useful biological and pharmacological properties. ${ }^{2}$ Phosphorus ylides are reactive intermediates, which take part in many valuable reactions in organic synthesis, ${ }^{3-5}$ and several methods have been developed for their preparation. These ylides are usually prepared by treatment of a phosphonium salt with a base, and phosphonium salts are usually prepared from the phosphine and an alkyl halide. ${ }^{1,6-10}$ Among other methods, phosphonium salts are also prepared by Michael addition of phosphorus nucleophiles to activated olefins. ${ }^{6-21}$ We report here an efficient synthetic route for the generation of stable phosphoranes bearing cyano groups via the reaction of triphenylphosphine with dialkyl acetylenedicarboxylates (1) in the presence of 
strong $\mathrm{CH}$-acids such as malononitrile or ethyl cyanoacetate (2), which leads to the corresponding stable phosphorus ylides (3) in excellent yields (Scheme 1).

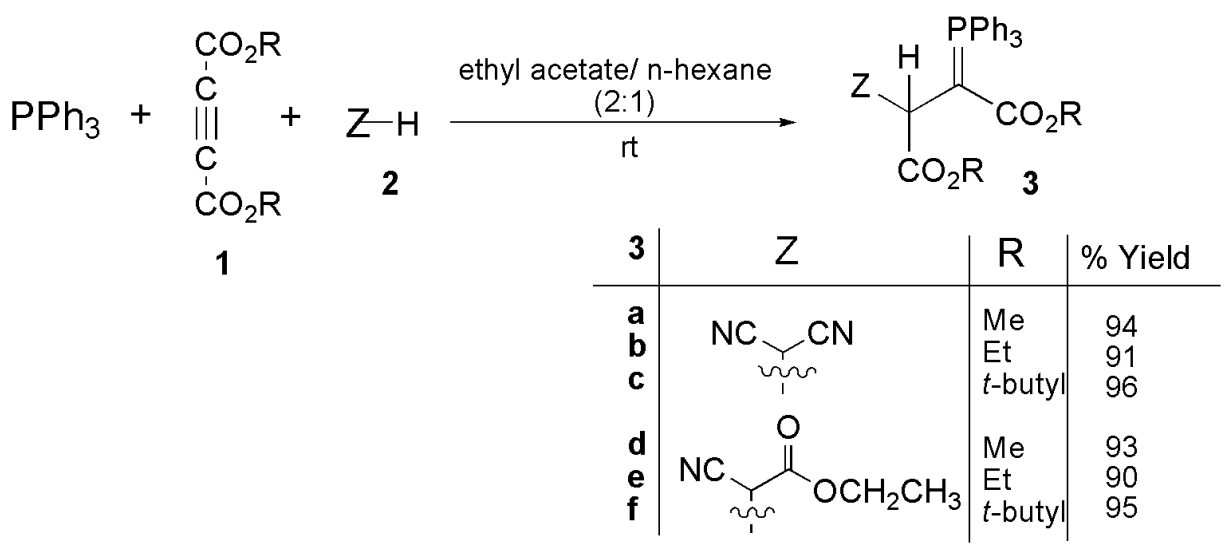

\section{Scheme 1}

\section{Result and Discussion}

The reactions of malononitrile or ethyl cyanoacetate (2) with dialkyl acetylenedicarboxylates (1) in the presence of triphenylphosphine proceeded in a mixture of ethyl acetate and $n$-hexane $(2: 1)$ at room temperature and were complete within 1 hour. The ${ }^{1} \mathrm{H}$ - and ${ }^{13} \mathrm{C}$ - NMR spectra of the crude product clearly indicated the formation of stable phosphorus ylides, 3 . No products other than 3 could be detected by NMR spectroscopy. The structures of compounds $\mathbf{3 a - f}$ were deduced from their IR-, ${ }^{1} \mathrm{H}-,{ }^{13} \mathrm{C}$ - and ${ }^{31} \mathrm{P}$ - NMR spectra. The mass spectra of the products displayed molecular ion peaks at appropriate $\mathrm{m} / \mathrm{z}$ values. Any initial fragmentations involved the loss of alkoxy, alkoxycarbonyl, and $\mathrm{PPh}_{3}$ moieties.

The ${ }^{1} \mathrm{H}-,{ }^{13} \mathrm{C}$-, and ${ }^{31} \mathrm{P}$ - NMR spectra of ylides $3 \mathbf{a}-\mathbf{f}$ are consistent with the presence of two geometrical isomers. The ylide moiety of these compounds is conjugated with the adjacent carbonyl group, and rotation around the partial double bond of the (E)- $\mathbf{3}$ and (Z)- $\mathbf{3}$ geometrical isomers is slow on the NMR timescale at ambient temperature. Selected ${ }^{1} \mathrm{H}-,{ }^{13} \mathrm{C}-$, and ${ }^{31} \mathrm{P}-\mathrm{NMR}$ chemical shifts and coupling constants in the major $(\mathrm{M})$ and minor $(\mathrm{m})$ geometrical isomers of compounds 3a-f are shown in Table 1. On the basis of the well-established chemistry of trivalent phosphorus nucleophiles, ${ }^{1,6,7}$ it is reasonable to assume that the phosphorus ylides (3) result from the initial addition of triphenylphosphine to the acetylenic ester and subsequent deprotonation of the 1:1 adduct (Scheme 2). 


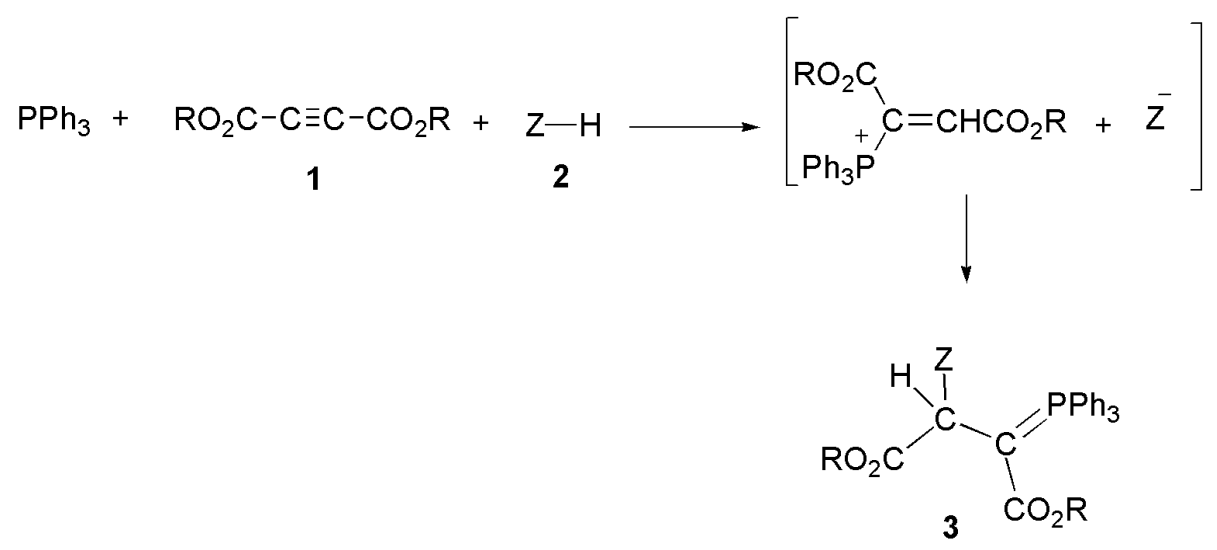

\section{Scheme 2}
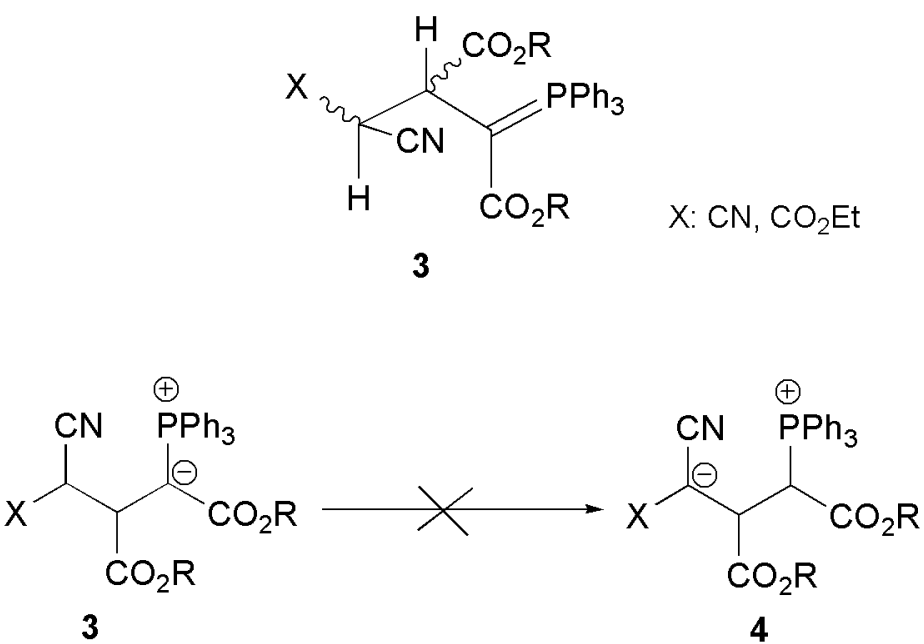

\section{Scheme 3}

The ${ }^{1} \mathrm{H}$ - NMR spectra of compounds $\mathbf{3}$ verified that compound $\mathbf{4}$ had not been formed under these conditions, and the anti-conformation of the two vicinal protons in compounds 3 was supported by the value of ${ }^{3} J_{\mathrm{HH}}$ : partial assignments are given in the Experimental Section (see Scheme 3 and Table 1). 
Table 1. Selected ${ }^{1} \mathrm{H}-,{ }^{13} \mathrm{C}$-, and ${ }^{31} \mathrm{P}$ - NMR chemical shifts ( $\delta$ in ppm) and coupling constants $(J$ in $\mathrm{Hz}$ ) for $\mathrm{H}-2,-\mathrm{OR},-\mathrm{CO}_{2} \mathrm{R}, \mathrm{C}-2$, and $\mathrm{C}-3$, in the Major (M) and Minor (m) diastereoisomers of compounds 3a-f.<smiles>[R]OC(=O)[Z]([H])([H])[C@H]([Z])C([PH3+])=C([O-])O[R]</smiles>

3 -(Z); Miajor

$3-(E)$; Minor

\begin{tabular}{|c|c|c|c|c|c|c|c|}
\hline \multirow[b]{2}{*}{ Compd } & \multirow[b]{2}{*}{$\begin{array}{c}\text { Isomer } \\
(\%)\end{array}$} & \multicolumn{3}{|c|}{${ }^{1} \mathrm{H}$ NMR data } & \multicolumn{3}{|c|}{${ }^{13} \mathrm{C}$ NMR data } \\
\hline & & $\begin{array}{c}\mathrm{H}-2 \\
\left({ }^{3} J_{\mathrm{PH}}\right)\end{array}$ & OR & $\mathrm{CO}_{2} \mathrm{R}$ & $\begin{array}{c}\mathrm{C}-2 \\
\left({ }^{2} J_{\mathrm{PC}}\right)\end{array}$ & $\begin{array}{c}\mathrm{C}-3 \\
\left({ }^{1} J_{\mathrm{PC}}\right)\end{array}$ & $\begin{array}{l}{ }^{31} \mathrm{P} \\
\mathrm{NMR}\end{array}$ \\
\hline 3a & M (74) & $\begin{array}{c}2.90 \\
(16.0)\end{array}$ & 3.17 & 3.77 & $\begin{array}{l}46.88 \\
(14.5)\end{array}$ & $\begin{array}{c}39.66 \\
(128.8)\end{array}$ & 22.90 \\
\hline 3a & m (26) & $\begin{array}{c}2.95 \\
(15.6)\end{array}$ & 3.58 & 3.76 & $\begin{array}{l}46.20 \\
(14.2)\end{array}$ & $\begin{array}{c}40.75 \\
(137.3)\end{array}$ & 23.60 \\
\hline $3 \mathbf{b}$ & M (75) & $\begin{array}{c}2.88 \\
(16.1)\end{array}$ & 3.71 & 4.25 & $\begin{array}{l}46.92 \\
(15.1)\end{array}$ & $\begin{array}{c}39.56 \\
(129.1)\end{array}$ & 22.80 \\
\hline $3 \mathbf{b}$ & $\mathrm{m}(25)$ & $\begin{array}{c}2.94 \\
(17.7)\end{array}$ & 4.13 & 4.31 & $\begin{array}{l}46.39 \\
(14.2)\end{array}$ & $\begin{array}{c}40.83 \\
(137.4)\end{array}$ & 23.61 \\
\hline $3 c$ & M (79) & $\begin{array}{c}2.74 \\
(16.2)\end{array}$ & 0.95 & 1.50 & $\begin{array}{l}47.83 \\
(15.1)\end{array}$ & $\begin{array}{c}39.54 \\
(129.8)\end{array}$ & 22.40 \\
\hline 3c & $\mathrm{m}(21)$ & $\begin{array}{c}2.71 \\
(16.3)\end{array}$ & 1.45 & 1.47 & $\begin{array}{l}46.93 \\
(14.7)\end{array}$ & $\begin{array}{c}41.31 \\
(137.5)\end{array}$ & 23.87 \\
\hline 3d & M (64) & $\begin{array}{c}3.05 \\
(16.6)\end{array}$ & 3.14 & 3.65 & $\begin{array}{l}44.37 \\
(14.4)\end{array}$ & $\begin{array}{c}39.39 \\
(128.3)\end{array}$ & 23.20 \\
\hline 3d & m (36) & $\begin{array}{c}3.10 \\
(16.5)\end{array}$ & 3.56 & 3.64 & $\begin{array}{l}43.62 \\
(14.5)\end{array}$ & $\begin{array}{c}40.31 \\
(136.9)\end{array}$ & 23.57 \\
\hline $3 \mathbf{e}$ & M (67) & $\begin{array}{c}3.05 \\
(16.5)\end{array}$ & $3.05-3.11$ & $4.00-4.20$ & $\begin{array}{l}44.47 \\
(14.5)\end{array}$ & $\begin{array}{c}39.27 \\
(128.5)\end{array}$ & 23.19 \\
\hline $3 \mathbf{e}$ & m (33) & $\begin{array}{c}3.11 \\
(16.6)\end{array}$ & $3.71-3.78$ & $4.00-4.20$ & $\begin{array}{l}43.84 \\
(14.3)\end{array}$ & $\begin{array}{c}40.44 \\
(136.9)\end{array}$ & 23.73 \\
\hline $3 f$ & M (70) & $\begin{array}{c}2.84 \\
(18.7)\end{array}$ & 0.95 & 1.43 & $\begin{array}{l}45.37 \\
(14.8)\end{array}$ & $\begin{array}{c}39.07 \\
(129.1)\end{array}$ & 22.56 \\
\hline $3 f$ & $\mathrm{~m}(30)$ & $\begin{array}{c}2.91 \\
(17.1)\end{array}$ & 1.41 & 1.45 & $\begin{array}{l}44.28 \\
(14.7)\end{array}$ & $\begin{array}{c}41.01 \\
(136.3)\end{array}$ & 22.40 \\
\hline
\end{tabular}


The assignment of 3-(E)- and 3-(Z)- rotamers as the major and minor components in phosphorus ylides have been reported previously in the literature. ${ }^{22-24} \mathrm{The}{ }^{1} \mathrm{H}$ - NMR spectrum of compound 3a exhibits two sharp lines ( $\delta 3.17$ and 3.77 ) arising from the methoxy group in the Zrotamer and two single sharp resonances at 3.58 and $3.76 \mathrm{ppm}$ for methoxy groups in the Erotamer. The shift at 3.21 of the methoxy group of the $Z$ - rotamer is shielded, due to the anisotropic effect of the phenyl groups of triphenylphosphine. This effect confirms why the 3-E and $3-Z$ rotamers could appear as the minor and major forms, respectively, as reported in the Experimental Section. Also, the signals for methine protons at $\delta 2.90,2.95,4.91$ and $5.46 \mathrm{ppm}$, appear as two doublets of doublets $\left({ }^{3} J_{\mathrm{PH}}=16\right.$ and $\left.{ }^{3} J_{\mathrm{HH}}=10.5 \mathrm{~Hz}\right)$ and $\left({ }^{3} J_{\mathrm{PH}}=15.6\right.$ and ${ }^{3} J_{\mathrm{HH}}=10.5$ $\mathrm{Hz})$ and two doublets $\left({ }^{3} \mathrm{JHH}_{\mathrm{HH}}=10.5\right.$ and $\left.{ }^{3} \mathrm{~J}_{\mathrm{HH}}=10.5 \mathrm{~Hz}\right)$ for the major and minor geometrical isomers, respectively. The ${ }^{13} \mathrm{C}$ - NMR spectrum of $3 \mathbf{a}$ exhibits 26 distinct resonances, which are in agreement with the mixture of two conformational isomers. Although the presence of the ${ }^{31} \mathrm{P}$ nucleus complicated both the ${ }^{1} \mathrm{H}$ - and ${ }^{13} \mathrm{C}$ - NMR spectra of phosphorane 3a, it helps in assigning the signals by long-range spin-spin couplings with the ${ }^{1} \mathrm{H}$ - and ${ }^{13} \mathrm{C}$ - nuclei. The ${ }^{1} \mathrm{H}$ - and ${ }^{13} \mathrm{C}-$ NMR spectra of compounds $\mathbf{3 b}-\mathbf{c}$ are similar to those of 3a, except for the signals from the ester group, which appear as characteristic resonance lines with the corresponding chemical shifts. The structural assignments made for phosphoranes 3a-f on the basis of the ${ }^{1} \mathrm{H}$ - and ${ }^{13} \mathrm{C}$ - NMR spectra were supported by their IR spectra. Of special interest is the absorption of the ester groups of such compounds at $1742-1614 \mathrm{~cm}^{-1}$. The conjugation of one ester group with the negative charge is a plausible factor in the decrease in the wavenumbers of the corresponding carbonyl absorption bands.

In summary, we have prepared novel stable cyano-functional phosphorus ylides using a onepot reaction between triphenylphosphine and dialkyl acetylenedicarboxylates in the presence of strong $\mathrm{CH}$-acids such as malononitrile or ethyl cyanoacetate. The present method has the advantage that not only is the reaction performed under neutral conditions, but also that the reagents can be mixed without any activation or modification.

\section{Experimental Section}

General Procedures. Melting points and IR spectra of all compounds were measured on an Electrothermal 9100 apparatus and a Shimadzu IR-460 spectrometer respectively. The ${ }^{1} \mathrm{H}-,{ }^{13} \mathrm{C}-$, and ${ }^{31} \mathrm{P}$ - NMR spectra were obtained from a BRUKER DRX-300 AVANCE instrument with $\mathrm{CDCl}_{3}$ as a solvent at $300.1,75.5$, and $121.5 \mathrm{MHz}$, respectively. Elemental analyses for $\mathrm{C}, \mathrm{H}$, and $\mathrm{N}$ were performed using a Heraeus CHN-O-Rapid analyzer. Mass spectra were recorded on Shimadzu GCMS-QP5050A and Finnigan-MAT 8430 mass spectrometers operating at an ionization potential of $70 \mathrm{eV}$. Dialkyl acetylenedicarboxylates, triphenylphosphine, malononitrile, and ethyl cyanoacetate were purchased from Fluka (Buchs) and used without further purification. RT denotes room temperature. 


\section{General preparative procedure, exemplified by dimethyl 2-(dicyanomethyl)-3-} (triphenylphosphanylidene)butanedioate (3a)

To a magnetically stirred solution of malononitrile $(0.06 \mathrm{~g}, 1 \mathrm{mmol})$ and triphenylphosphine $(0.26 \mathrm{~g}, 1 \mathrm{mmol})$ in a mixture of ethyl acetate and $n$-hexane $(2: 1, \mathrm{v} / \mathrm{v})$ was added dropwise a mixture of dimethyl acetylenedicarboxylate $(0.12 \mathrm{~mL}, 1 \mathrm{mmol})$ in $3 \mathrm{~mL}$ of ethyl acetate at $-5{ }^{\circ} \mathrm{C}$ over $10 \mathrm{~min}$. After approximately 1 hour stirring at RT the product was filtered off and washed with cold diethyl ether $(3 \times 5 \mathrm{~mL})$, and was finally obtained as a white powder, $0.44 \mathrm{~g}$, yield 94\%, mp 182-185 ${ }^{\circ} \mathrm{C}$, IR $(\mathrm{KBr})\left(v_{\max } \mathrm{cm}^{-1}\right): 2225(\mathrm{CN}), 1735$ and $1618(\mathrm{C}=\mathrm{O})$. MS $(\mathrm{m} / \mathrm{z}, \%)$ : 406 (30), 405 (100), 262 (8), 183 (27), 108 (15), 77 (13), 59 (8). Anal. Calcd for $\mathrm{C}_{27} \mathrm{H}_{23} \mathrm{~N}_{2} \mathrm{O}_{4} \mathrm{P}$ (470.46): C, 68.93; H, 4.93; N, 5.95. Found: $\mathrm{C}, 69.04 ; \mathrm{H}, 4.93 ; \mathrm{N}, 6.02 \%$. Major isomer (Z)-3a (74\%): ${ }^{1} \mathrm{H}$ NMR (300.1 MHz): $\delta 2.90\left(1 \mathrm{H}, \mathrm{dd},{ }^{3} \mathrm{~J}_{\mathrm{HH}}=10.5 \mathrm{~Hz},{ }^{3} \mathrm{~J}_{\mathrm{PH}}=16.0 \mathrm{~Hz}, \mathrm{P}=\mathrm{C}-\mathrm{CH}\right), 3.17$ and $3.77\left(6 \mathrm{H}, 2 \mathrm{~s}, 2 \mathrm{xOCH}_{3}\right), 5.46\left(1 \mathrm{H}, \mathrm{d},{ }^{3} J_{\mathrm{HH}}=10.5 \mathrm{~Hz}, \mathrm{CH}(\mathrm{CN})_{2}\right), 7.52-7.77\left(15 \mathrm{H}, \mathrm{m}, 3 \mathrm{xC}_{6} \mathrm{H}_{5}\right) .{ }^{13} \mathrm{C}$ NMR (75.5 MHz): $\delta 24.80\left(\mathrm{~d},{ }^{3} J_{\mathrm{PC}}=4.9 \mathrm{~Hz}, \mathrm{CH}(\mathrm{CN})_{2}\right), 39.66\left(\mathrm{~d},{ }^{1} J_{\mathrm{PC}}=128.8 \mathrm{~Hz}, \mathrm{P}=C\right), 46.88(\mathrm{~d}$, $\left.{ }^{2} J_{\mathrm{PC}}=14.5 \mathrm{~Hz}, \mathrm{P}=\mathrm{C}-\mathrm{CH}\right), 49.34$ and $52.61\left(2 \mathrm{~s}, 2 \mathrm{xOCH}_{3}\right), 113.86$ and $113.90(2 \mathrm{~s}, 2 \mathrm{xCN}), 125.64$ $\left(\mathrm{d},{ }^{1} J_{\mathrm{PC}}=92.8 \mathrm{~Hz}, \mathrm{C}_{\mathrm{ipso}}\right), 128.91\left(\mathrm{~d},{ }^{3} J_{\mathrm{PC}}=12.3 \mathrm{~Hz}, \mathrm{C}_{\text {meta }}\right), 132.63\left(\mathrm{~d},{ }^{4} J_{\mathrm{PC}}=2.6 \mathrm{~Hz}, \mathrm{C}_{\mathrm{para}}\right), 139.91$ $\left(\mathrm{d},{ }^{2} J_{\mathrm{PC}}=9.8 \mathrm{~Hz}, \mathrm{C}_{\mathrm{ortho}}\right), 169.34\left(\mathrm{~d},{ }^{2} J_{\mathrm{PC}}=12.3 \mathrm{~Hz}, \mathrm{P}-\mathrm{C}=\mathrm{C}\right), 171.11\left(\mathrm{~d},{ }^{3} \mathrm{JCC}_{\mathrm{PC}}=4.0 \mathrm{~Hz}, \mathrm{C}=\mathrm{O}\right.$, ester). ${ }^{31} \mathrm{P}$ NMR (121.5 MHz): $\delta 22.90\left(\mathrm{Ph}_{3} \mathrm{P}^{+}-\mathrm{C}\right)$. Minor isomer $(E)-3 \mathrm{a}(26 \%):{ }^{1} \mathrm{H}$ NMR $(300.1 \mathrm{MHz})$ : $\delta 2.95\left(1 \mathrm{H}, \mathrm{dd},{ }^{3} J_{\mathrm{HH}}=10.5 \mathrm{~Hz},{ }^{3} J_{\mathrm{PH}}=15.6 \mathrm{~Hz}, \mathrm{P}=\mathrm{C}-\mathrm{CH}\right), 3.58$ and $3.76\left(6 \mathrm{H}, 2 \mathrm{~s}, 2 \mathrm{xOCH}_{3}\right)$, 4.91 $\left(1 \mathrm{H}, \mathrm{d},{ }^{3} \mathrm{~J}_{\mathrm{HH}}=10.5 \mathrm{~Hz}, \mathrm{CH}(\mathrm{CN})_{2}\right), 7.52-7.77\left(15 \mathrm{H}, m, 3 \mathrm{xC}_{6} \mathrm{H}_{5}\right) .{ }^{13} \mathrm{C} \mathrm{NMR}(75.5 \mathrm{MHz}): \delta$ $26.37\left(\mathrm{~d},{ }^{3} J_{\mathrm{PC}}=5.3 \mathrm{~Hz}, \mathrm{CH}(\mathrm{CN})_{2}\right), 40.75\left(\mathrm{~d},{ }^{1} J_{\mathrm{PC}}=137.3 \mathrm{~Hz}, \mathrm{P}=C\right), 46.20\left(\mathrm{~d},{ }^{2} J_{\mathrm{PC}}=14.2 \mathrm{~Hz}, \mathrm{P}=\mathrm{C}-\right.$ $\mathrm{CH}), 50.35$ and $52.58\left(2 \mathrm{~s}, 2 \mathrm{xOCH}_{3}\right), 113.53$ and $113.63(2 \mathrm{~s}, 2 \mathrm{xCN}), 125.13\left(\mathrm{~d},{ }^{1} \mathrm{~J}_{\mathrm{PC}}=92.9 \mathrm{~Hz}\right.$, $\left.\mathrm{C}_{\mathrm{ipso}}\right), 128.98\left(\mathrm{~d},{ }^{3} J_{\mathrm{PC}}=12.3 \mathrm{~Hz}, \mathrm{C}_{\text {meta }}\right), 132.63\left(\mathrm{~d},{ }^{4} J_{\mathrm{PC}}=2.6 \mathrm{~Hz}, \mathrm{C}_{\mathrm{para}}\right), 133.96\left(\mathrm{~d},{ }^{2} J_{\mathrm{PC}}=9.8 \mathrm{~Hz}\right.$, $\left.\mathrm{C}_{\text {ortho }}\right), 169.59\left(\mathrm{~d},{ }^{2} J_{\mathrm{PC}}=16.1 \mathrm{~Hz}, \mathrm{P}-\mathrm{C}=\mathrm{C}\right), 171.48\left(\mathrm{~d},{ }^{3} J_{\mathrm{PC}}=4.8 \mathrm{~Hz}, \mathrm{C}=\mathrm{O}\right.$, ester $) .{ }^{31} \mathrm{P}$ NMR $(121.5$ $\mathrm{MHz}): \delta 23.60\left(\mathrm{Ph}_{3} \mathrm{P}^{+}-\mathrm{C}\right)$.

Diethyl 2-(dicyanomethyl)-3-(triphenylphosphanylidene)butanedioate (3b). The yield was $0.45 \mathrm{~g}, 91 \%$, mp $169-172{ }^{\circ} \mathrm{C}$, IR (KBr) $\left(v_{\max }, \mathrm{cm}^{-1}\right): 2250(\mathrm{CN}), 1727$ and $1620(\mathrm{C}=\mathrm{O})$. MS $(\mathrm{m} / \mathrm{z}$, \%): 453 (M+OEt, 5), 434 (9), 433 (100), 425 (7), 262 (11), 183 (39), 108 (18), 77 (13). Anal. Calcd for $\mathrm{C}_{29} \mathrm{H}_{27} \mathrm{~N}_{2} \mathrm{O}_{4} \mathrm{P}$ (498.51): C, 69.87; H, 5.46; N, 5.62. Found: C, 69.80; H, 5.49; N, $5.58 \%$. Major isomer (Z)-3b (75\%): ${ }^{1} \mathrm{H}$ NMR (300.1 MHz): $\delta 0.47$ and $1.26\left(6 \mathrm{H}, 2 \mathrm{t},{ }^{3} \mathrm{~J}_{\mathrm{HH}}=7.1\right.$ $\left.\mathrm{Hz}, 2 \mathrm{xOCH}_{2} \mathrm{CH}_{3}\right), 2.88\left(1 \mathrm{H}, \mathrm{dd},{ }^{3} J_{\mathrm{HH}}=10.5 \mathrm{~Hz},{ }^{3} \mathrm{JH}_{\mathrm{PH}}=16.1 \mathrm{~Hz}, \mathrm{P}=\mathrm{C}-\mathrm{CH}\right), 3.71$ and $4.25(4 \mathrm{H}, 2 \mathrm{q}$, $\left.J=7.1 \mathrm{~Hz}, 2 \mathrm{xOCH}_{2} \mathrm{CH}_{3}\right), 5.45\left(1 \mathrm{H}, \mathrm{d},{ }^{3} J_{\mathrm{HH}}=10.5 \mathrm{~Hz}, \mathrm{CH}(\mathrm{CN})_{2}\right), 7.48-7.80\left(15 \mathrm{H}, \mathrm{m}, 3 \mathrm{xC}_{6} \mathrm{H}_{5}\right)$. ${ }^{13} \mathrm{C}$ NMR (75.5 MHz): $\delta 13.80$ and $14.06\left(2 \mathrm{~s}, 2 \mathrm{xCH}_{3}\right), 24.83\left(\mathrm{~d},{ }^{3} J_{\mathrm{PC}}=5.2 \mathrm{~Hz}, \mathrm{CH}(\mathrm{CN})_{2}\right), 39.56$ $\left(\mathrm{d},{ }^{1} J_{\mathrm{PC}}=129.1 \mathrm{~Hz}, \mathrm{P}=\mathrm{C}\right), 46.92\left(\mathrm{~d},{ }^{2} \mathrm{~J}_{\mathrm{PC}}=15.1 \mathrm{~Hz}, \mathrm{P}=\mathrm{C}-\mathrm{CH}\right), 58.02$ and $61.84\left(2 \mathrm{~s}, 2 \mathrm{xOCH}_{2} \mathrm{CH}_{3}\right)$, 114.06 and $114.03(2 \mathrm{~s}, 2 \mathrm{xCN}), 125.83\left(\mathrm{~d},{ }^{1} J_{\mathrm{PC}}=92.8 \mathrm{~Hz}, \mathrm{C}_{\mathrm{ipso}}\right), 128.82\left(\mathrm{~d},{ }^{3} J_{\mathrm{PC}}=12.1 \mathrm{~Hz}, \mathrm{C}_{\text {meta }}\right)$, $132.57\left(\mathrm{~d},{ }^{4} J_{\mathrm{PC}}=2.2 \mathrm{~Hz}, \mathrm{C}_{\mathrm{para}}\right), 134.0\left(\mathrm{~d},{ }^{2} J_{\mathrm{PC}}=9.7 \mathrm{~Hz}, \mathrm{C}_{\text {ortho }}\right), 168.95\left(\mathrm{~d},{ }^{2} J_{\mathrm{PC}}=12.1 \mathrm{~Hz}, \mathrm{P}-\mathrm{C}=C\right)$, $170.68\left(\mathrm{~d},{ }^{3} J_{\mathrm{PC}}=3.7 \mathrm{~Hz}, \mathrm{C}=\mathrm{O}\right.$, ester $) .{ }^{31} \mathrm{P}$ NMR $(121.5 \mathrm{MHz}): \delta 22.80\left(\mathrm{Ph}_{3} \mathrm{P}^{+}-\mathrm{C}\right)$. Minor isomer (E)-3b (25\%): ${ }^{1} \mathrm{H}$ NMR (300.1 MHz): $\delta 1.20$ and $1.30\left(6 \mathrm{H}, 2 \mathrm{t},{ }^{3} J_{\mathrm{HH}}=7.1 \mathrm{~Hz}, 2 \mathrm{xOCH}_{2} \mathrm{CH}_{3}\right), 2.94$ $\left(1 \mathrm{H}, \mathrm{dd},{ }^{3} J_{\mathrm{HH}}=10.3 \mathrm{~Hz},{ }^{3} J_{\mathrm{PH}}=17.7 \mathrm{~Hz}, \mathrm{P}=\mathrm{C}-\mathrm{CH}\right), 4.13$ and $4.31\left(4 \mathrm{H}, 2 \mathrm{~m}, 2 \mathrm{xABX}{ }_{3}\right.$ system, $\left.2 \mathrm{xOCH}_{2} \mathrm{CH}_{3}\right), 4.93\left(1 \mathrm{H}, \mathrm{d},{ }^{3} \mathrm{~J}_{\mathrm{HH}}=10.3 \mathrm{~Hz}, \mathrm{CH}(\mathrm{CN})_{2}\right), 7.48-7.80\left(15 \mathrm{H}, \mathrm{m}, 3 \mathrm{xC}_{6} \mathrm{H}_{5}\right) .{ }^{13} \mathrm{C} \mathrm{NMR}$ $(75.5 \mathrm{MHz}): \delta 14.14$ and $14.91\left(2 \mathrm{~s}, 2 \mathrm{xCH}_{3}\right), 26.40\left(\mathrm{~d},{ }^{3} \mathrm{~J}_{\mathrm{PC}}=5.2 \mathrm{~Hz}, \mathrm{CH}(\mathrm{CN})_{2}\right), 40.83(\mathrm{~d}$, 
$\left.{ }^{1} J_{\mathrm{PC}}=137.4 \mathrm{~Hz}, \mathrm{P}=C\right), 46.39\left(\mathrm{~d},{ }^{2} J_{\mathrm{PC}}=14.2 \mathrm{~Hz}, \mathrm{P}=\mathrm{C}-\mathrm{CH}\right), 58.62$ and $61.91\left(2 \mathrm{~s}, 2 \mathrm{xOCH}_{2} \mathrm{CH}_{3}\right)$, 113.75 and $113.64(2 \mathrm{~s}, 2 \mathrm{xCN}), 125.31\left(\mathrm{~d},{ }^{1} J_{\mathrm{PC}}=92.8 \mathrm{~Hz}, \mathrm{C}_{\mathrm{ipso}}\right), 128.91\left(\mathrm{~d},{ }^{3} J_{\mathrm{PC}}=12.1 \mathrm{~Hz}, \mathrm{C}_{\text {meta }}\right.$ ), $132.57\left(\mathrm{~d},{ }^{4} J_{\mathrm{PC}}=2.2 \mathrm{~Hz}, \mathrm{C}_{\mathrm{para}}\right), 134.0\left(\mathrm{~d},{ }^{2} J_{\mathrm{PC}}=9.7 \mathrm{~Hz}, \mathrm{C}_{\text {ortho }}\right), 169.20\left(\mathrm{~d},{ }^{2} J_{\mathrm{PC}}=17.3 \mathrm{~Hz}, \mathrm{P}-\mathrm{C}=C\right)$, $171.04\left(\mathrm{~d},{ }^{3} J_{\mathrm{PC}}=3.7 \mathrm{~Hz}, \mathrm{C}=\mathrm{O}\right.$, ester $) .{ }^{31} \mathrm{P}$ NMR $(202.4 \mathrm{MHz}): \delta 23.61\left(\mathrm{Ph}_{3} \mathrm{P}^{+}-\mathrm{C}\right)$.

Di- tert-butyl 2-(dicyanomethyl)-3-(triphenylphosphanylidene)butanedioate (3c). The yield was $0.53 \mathrm{~g}, 96 \%$, mp 158-160 ${ }^{\circ} \mathrm{C}$, IR $(\mathrm{KBr})\left(v_{\max }, \mathrm{cm}^{-1}\right): 2247(\mathrm{CN}), 1723$ and $1614(\mathrm{C}=\mathrm{O})$. MS (m/z, \%): $554\left(\mathrm{M}^{+}, 7\right), 490$ (15), 489 (35), 337 (100), 262 (22), 183 (38), 108 (17), 77 (8), 57 (75). Anal. Calcd for $\mathrm{C}_{33} \mathrm{H}_{35} \mathrm{~N}_{2} \mathrm{O}_{4} \mathrm{P}$ (554.62): C, 71.46; H, 6.36; N, 5.05. Found: C, 71.55; H, $6.37 ; \mathrm{N}, 4.98 \%$.

Major isomer (Z)-3c. (79\%): ${ }^{1} \mathrm{H}$ NMR (300.1 MHz), $\delta 0.95$ and $1.50(18 \mathrm{H}, 2 \mathrm{~s}, 2 \mathrm{x} \mathrm{CMe}), 2.74$ $\left(1 \mathrm{H}, \mathrm{dd},{ }^{3} J_{\mathrm{HH}}=10.4 \mathrm{~Hz},{ }^{3} J_{\mathrm{PH}}=16.2 \mathrm{~Hz}, \mathrm{P}=\mathrm{C}-\mathrm{CH}\right), 5.44\left(1 \mathrm{H}, \mathrm{d},{ }^{3} J_{\mathrm{HH}}=10.4 \mathrm{~Hz}, \mathrm{CH}(\mathrm{CN})_{2}\right), 7.52-$ $7.81\left(15 \mathrm{H}, \mathrm{m}, 3 \mathrm{xC}_{6} \mathrm{H}_{5}\right) .{ }^{13} \mathrm{C} \mathrm{NMR}(75.5 \mathrm{MHz}), \delta 24.91\left(\mathrm{~d},{ }^{3} \mathrm{~J}_{\mathrm{PC}}=4.6 \mathrm{~Hz}, \mathrm{CH}(\mathrm{CN})_{2}\right), 28.00$ and $28.22\left(2 \mathrm{~s}, 2 \mathrm{x} \mathrm{CMe}_{3}\right), 39.54\left(\mathrm{~d},{ }^{1} J_{\mathrm{PC}}=129.8 \mathrm{~Hz}, \mathrm{P}=\mathrm{C}\right), 47.83\left(\mathrm{~d},{ }^{2} J_{\mathrm{PC}}=15.1 \mathrm{~Hz}, \mathrm{P}=\mathrm{C}-\mathrm{CH}\right), 78.09$ and $82.32\left(2 \mathrm{~s}, \mathrm{OCMe}_{3}\right), 114.46$ and $114.27(2 \mathrm{~s}, 2 \mathrm{xCN}), 126.40\left(\mathrm{~d},{ }^{1} J_{\mathrm{PC}}=92.4 \mathrm{~Hz}, \mathrm{C}_{\mathrm{ipso}}\right), 128.61$ $\left(\mathrm{d},{ }^{3} J_{\mathrm{PC}}=12.1 \mathrm{~Hz}, \mathrm{C}_{\text {meta }}\right), 132.34\left(\mathrm{~d},{ }^{4} J_{\mathrm{PC}}=2.3 \mathrm{~Hz}, \mathrm{C}_{\mathrm{para}}\right), 134.12\left(\mathrm{~d},{ }^{2} J_{\mathrm{PC}}=9.7 \mathrm{~Hz}, \mathrm{C}_{\text {ortho }}\right), 168.48$ (d, $\left.{ }^{2} J_{\mathrm{PC}}=11.7 \mathrm{~Hz}, \mathrm{P}-\mathrm{C}=C\right), 169.78\left(\mathrm{~d},{ }^{3} J_{\mathrm{PC}}=4.5 \mathrm{~Hz}, \mathrm{C}=\mathrm{O}\right) .{ }^{31} \mathrm{P} \mathrm{NMR}(121.5 \mathrm{MHz}): \delta 22.40\left(\mathrm{Ph}_{3} \mathrm{P}^{+}\right.$C).

Minor isomer (E)-3c. (21\%): ${ }^{1} \mathrm{H}$ NMR (300.1 MHz), $\delta 1.45$ and $1.47(18 \mathrm{H}, 2 \mathrm{~s}, 2 \mathrm{x} \mathrm{CMe}), 2.71$ $\left(1 \mathrm{H}, \mathrm{dd},{ }^{3} J_{\mathrm{HH}}=10.4 \mathrm{~Hz},{ }^{3} J_{\mathrm{PH}}=16.3 \mathrm{~Hz}, \mathrm{P}=\mathrm{C}-\mathrm{CH}\right), 5.00\left(1 \mathrm{H}, \mathrm{d},{ }^{3} J_{\mathrm{HH}}=10.4 \mathrm{~Hz}, \mathrm{CH}(\mathrm{CN})_{2}\right), 7.52-$ $7.81\left(15 \mathrm{H}, \mathrm{m}, 3 \mathrm{xC}_{6} \mathrm{H}_{5}\right) .{ }^{13} \mathrm{C} \mathrm{NMR}(75.5 \mathrm{MHz}), \delta 27.76$ and $29.60\left(2 \mathrm{~s}, 2 \mathrm{CMe}_{3}\right), 25.00(\mathrm{~d}$, $\left.{ }^{3} J_{\mathrm{PC}}=5.3 \mathrm{~Hz}, \mathrm{CH}(\mathrm{CN})_{2}\right), 41.31\left(\mathrm{~d},{ }^{1} J_{\mathrm{PC}}=137.5 \mathrm{~Hz}, \mathrm{P}=\mathrm{C}\right), 46.93\left(\mathrm{~d},{ }^{2} J_{\mathrm{PC}}=14.7 \mathrm{~Hz}, \mathrm{P}=\mathrm{C}-\mathrm{CH}\right), 78.49$ and $82.30\left(2 \mathrm{~s}, \mathrm{OCMe}_{3}\right), 113.82$ and $113.98(2 \mathrm{~s}, 2 \mathrm{CN}), 125.80\left(\mathrm{~d},{ }^{1} \mathrm{~J}_{\mathrm{PC}}=92.7 \mathrm{~Hz}, \mathrm{C}_{\mathrm{ipso}}\right), 128.77$ $\left(\mathrm{d},{ }^{3} J_{\mathrm{PC}}=11.2 \mathrm{~Hz}, \mathrm{C}_{\text {meta }}\right), 132.34\left(\mathrm{~d},{ }^{4} J_{\mathrm{PC}}=2.3 \mathrm{~Hz}, \mathrm{C}_{\mathrm{para}}\right), 133.99\left(\mathrm{~d},{ }^{2} J_{\mathrm{PC}}=9.6 \mathrm{~Hz}, \mathrm{C}_{\text {ortho }}\right), 168.50$ (d, $\left.{ }^{2} J_{\mathrm{PC}}=11.1 \mathrm{~Hz}, \mathrm{P}-\mathrm{C}=C\right), 169.56\left(\mathrm{~d},{ }^{3} J_{\mathrm{PC}}=4.9 \mathrm{~Hz}, \mathrm{C}=\mathrm{O}\right) .{ }^{31} \mathrm{P} \mathrm{NMR}(121.5 \mathrm{MHz}): \delta 23.87\left(\mathrm{Ph}_{3} \mathrm{P}^{+}-\right.$ C).

Dimethyl 2-(ethyl-1-cyanoethanoat-1-yl)-3-(triphenylphosphanylidene)butanedioate (3d). The yield was $0.48 \mathrm{~g}, 93 \%$, mp 143-145 ${ }^{\circ} \mathrm{C}$, IR $(\mathrm{KBr})\left(v_{\max }, \mathrm{cm}^{-1}\right): 2235(\mathrm{CN}), 1736$ and 1621 $(\mathrm{C}=\mathrm{O})$. MS (m/z, \%): $517\left(\mathrm{M}^{+}, 4\right), 416$ (20), 404 (22), 262 (100), 187 (72), 108 (15). Anal. Calcd for $\mathrm{C}_{29} \mathrm{H}_{28} \mathrm{NO}_{6} \mathrm{P}$ (517.51): C, 67.31; H, 5.45; N, 2.71. Found: C, 67.39; H, 5.48; N, 2.65\%.

Major isomer (Z)-3d. (64\%): ${ }^{1} \mathrm{H} \mathrm{NMR}(300.1 \mathrm{MHz}): \delta 1.21\left(3 \mathrm{H}, \mathrm{t},{ }^{3} J_{\mathrm{HH}}=7.1 \mathrm{~Hz}, \mathrm{OCH}_{2} \mathrm{CH}_{3}\right)$, $3.05\left(1 \mathrm{H}, \mathrm{dd},{ }^{3} \mathrm{~J}_{\mathrm{HH}}=10.9 \mathrm{~Hz},{ }^{3} J_{\mathrm{PH}}=16.6 \mathrm{~Hz}, \mathrm{P}=\mathrm{C}-\mathrm{CH}\right), 3.14$ and $3.65\left(6 \mathrm{H}, 2 \mathrm{~s}, 2 \mathrm{xOCH}_{3}\right), 4.13(2 \mathrm{H}$, $\mathrm{m}, \mathrm{ABX}_{3}$ system, $\left.\mathrm{OCH}_{2} \mathrm{CH}_{3}\right), 5.02\left(1 \mathrm{H}, \mathrm{d},{ }^{3} J_{\mathrm{HH}}=10.9 \mathrm{~Hz}, \mathrm{CH}\left(\mathrm{CNCO}_{2} \mathrm{Et}\right)\right), 7.50-7.79\left(15 \mathrm{H}_{\text {arom, }}\right.$ $\left.\mathrm{m}, 3 \mathrm{xC}_{6} \mathrm{H}_{5}\right) .{ }^{13} \mathrm{C}$ NMR $(75.5 \mathrm{MHz}): \delta 13.85\left(\mathrm{~s}, \mathrm{OCH}_{2} \mathrm{CH}_{3}\right), 39.27 \quad\left(\mathrm{~d},{ }^{3} J_{\mathrm{PC}}=3.9 \mathrm{~Hz}\right.$, $\left.\mathrm{CH}\left(\mathrm{CNCO}_{2} \mathrm{Et}\right)\right), 39.39\left(\mathrm{~d},{ }^{1} J_{\mathrm{PC}}=128.3 \mathrm{~Hz}, \mathrm{P}=\mathrm{C}\right), 44.37\left(\mathrm{~d},{ }^{2} J_{\mathrm{PC}}=14.41 \mathrm{~Hz}, \mathrm{P}=\mathrm{C}-\mathrm{CH}\right), 49.10$ and $52.15\left(2 \mathrm{~s}, 2 \mathrm{xOCH}_{3}\right), 62.23\left(\mathrm{~s}, \mathrm{OCH}_{2}\right), 117.32(\mathrm{~s}, \mathrm{CN}), 126.39\left(\mathrm{~d},{ }^{1} J_{\mathrm{PC}}=92.4 \mathrm{~Hz}, \mathrm{C}_{\text {ipso }}\right), 128.68(\mathrm{~d}$, $\left.{ }^{3} J_{\mathrm{PC}}=12.3 \mathrm{~Hz}, \mathrm{C}_{\text {meta }}\right), 132.25\left(\mathrm{~d},{ }^{4} J_{\mathrm{PC}}=2.8 \mathrm{~Hz}, \mathrm{C}_{\mathrm{para}}\right), 134.03\left(\mathrm{~d},{ }^{2} J_{\mathrm{PC}}=9.7 \mathrm{~Hz}, \mathrm{C}_{\text {ortho }}\right), 166.26(\mathrm{~s}$, $\left.\mathrm{C}=\mathrm{O}, \mathrm{CO}_{2} \mathrm{Et}\right), 169.54\left(\mathrm{~d},{ }^{2} J_{\mathrm{PC}}=12.8 \mathrm{~Hz}, \mathrm{P}-\mathrm{C}=\mathrm{C}\right), 173.24\left(\mathrm{~d},{ }^{3} J_{\mathrm{PC}}=4.6 \mathrm{~Hz}, \mathrm{C}=\mathrm{O}\right) .{ }^{31} \mathrm{P}$ NMR $(202.4$ $\mathrm{MHz}): \delta 23.20\left(\mathrm{Ph}_{3} \mathrm{P}^{+}-\mathrm{C}\right)$.

Minor isomer (E)-3d. (36\%): ${ }^{1} \mathrm{H}$ NMR (300.1 MHz): $\delta 1.23\left(3 \mathrm{H}, \mathrm{t},{ }^{3} J_{\mathrm{HH}}=7.1 \mathrm{~Hz}, \mathrm{OCH}_{2} \mathrm{CH}_{3}\right)$, $3.10\left(1 \mathrm{H}, \mathrm{dd},{ }^{3} \mathrm{~J}_{\mathrm{HH}}=11.2 \mathrm{~Hz},{ }^{3} \mathrm{~J}_{\mathrm{PH}}=16.5 \mathrm{~Hz}, \mathrm{P}=\mathrm{C}-\mathrm{CH}\right), 3.56$ and $3.64\left(6 \mathrm{H}, 2 \mathrm{~s}, 2 \mathrm{xOCH}_{3}\right), 4.13(2 \mathrm{H}$, 
m, $\mathrm{ABX}_{3}$ system, $\left.\mathrm{OCH}_{2} \mathrm{CH}_{3}\right), 4.63\left(1 \mathrm{H}, \mathrm{d},{ }^{3} \mathrm{JHH}_{\mathrm{HH}}=11.2 \mathrm{~Hz}, \mathrm{CH}\left(\mathrm{CNCO}_{2} \mathrm{Et}\right)\right), 7.50-7.79(15 \mathrm{H}, \mathrm{m}$, $\left.3 \mathrm{xC}_{6} \mathrm{H}_{5}\right) .{ }^{13} \mathrm{C}$ NMR (75.5 MHz): $\delta 13.85\left(\mathrm{~s}, \mathrm{OCH}_{2} \mathrm{CH}_{3}\right), 40.75\left(\mathrm{~d},{ }^{3} \mathrm{JC}_{\mathrm{PC}}=3.9 \mathrm{~Hz}, \mathrm{CH}\left(\mathrm{CNCO}_{2} \mathrm{Et}\right)\right)$, $40.31\left(\mathrm{~d},{ }^{1} J_{\mathrm{PC}}=136.9 \mathrm{~Hz}, \mathrm{P}=\mathrm{C}\right), 43.62\left(\mathrm{~d},{ }^{2} J_{\mathrm{PC}}=14.5 \mathrm{~Hz}, \mathrm{P}=\mathrm{C}-\mathrm{CH}\right), 50.23$ and $52.15(2 \mathrm{~s}$, $\left.2 \mathrm{xOCH}_{3}\right), 62.37\left(\mathrm{~s}, \mathrm{OCH}_{2} \mathrm{CH}_{3}\right), 117.04(\mathrm{~s}, \mathrm{CN}), 125.80\left(\mathrm{~d},{ }^{1} J_{\mathrm{PC}}=92.5 \mathrm{~Hz}, \mathrm{C}_{\mathrm{ipso}}\right), 128.76(\mathrm{~d}$, $\left.{ }^{3} J_{\mathrm{PC}}=12.4 \mathrm{~Hz}, \mathrm{C}_{\text {meta }}\right), 132.25\left(\mathrm{~d},{ }^{4} J_{\mathrm{PC}}=2.8 \mathrm{~Hz}, \mathrm{C}_{\mathrm{para}}\right), 134.05\left(\mathrm{~d},{ }^{2} J_{\mathrm{PC}}=9.7 \mathrm{~Hz}, \mathrm{C}_{\text {ortho }}\right), 166.02$ (s, $\left.\mathrm{C}=\mathrm{O}, \mathrm{CO}_{2} \mathrm{Et}\right), 170.23\left(\mathrm{~d},{ }^{2} J_{\mathrm{PC}}=17.8 \mathrm{~Hz}, \mathrm{P}-\mathrm{C}=C\right), 173.69\left(\mathrm{~d},{ }^{3} J_{\mathrm{PC}}=5.1 \mathrm{~Hz}, \mathrm{C}=\mathrm{O}\right) .{ }^{31} \mathrm{P}$ NMR $(121.5$ $\mathrm{MHz}): \delta 23.57\left(\mathrm{Ph}_{3} \mathrm{P}^{+}-\mathrm{C}\right)$.

Diethyl 2-(ethyl-1-cyanoethanoat-1-yl)-3-(triphenylphosphanylidene)butanedioate (3e). The yield was $0.49 \mathrm{~g}, 90 \%, \mathrm{mp} 120-123{ }^{\circ} \mathrm{C}$, IR $(\mathrm{KBr})\left(v_{\max }, \mathrm{cm}^{-1}\right)$ : $2230(\mathrm{CN}), 1742,1725$ and 1619 $(\mathrm{C}=\mathrm{O})$. MS (m/z, \%): $546\left(\mathrm{M}^{+}+1,2\right), 534$ (15), 522 (23), 262 (65), 187 (79), 108 (100). Anal. Calcd for $\mathrm{C}_{31} \mathrm{H}_{32} \mathrm{NO}_{6} \mathrm{P}(545.56)$ : C, 68.25; H, 5.91; N, 2.57. Found: C, 68.57; H, 5.79; N, 2.65\%. Major isomer (Z)-3e. (67\%): ${ }^{1} \mathrm{H}$ NMR (300.1 MHz): $\delta 0.46\left(3 \mathrm{H}, \mathrm{t},{ }^{3} \mathrm{~J}_{\mathrm{HH}}=7.0 \mathrm{~Hz}, \mathrm{OCH}_{2} \mathrm{CH}_{3}\right)$, 1.17-1.24 (6H, m, 2xCH $), 3.05\left(1 \mathrm{H}, \mathrm{dd},{ }^{3} \mathrm{~J}_{\mathrm{HH}}=10.1 \mathrm{~Hz},{ }^{3} \mathrm{~J}_{\mathrm{PH}}=16.5 \mathrm{~Hz}, \mathrm{P}=\mathrm{C}-\mathrm{CH}\right), 3.05-3.11$ and 4.00-4.20 (6H, 2m, 3xABX 3 system, 3xOCH $\left.\mathrm{CH}_{3}\right), 5.01\left(1 \mathrm{H}, \mathrm{d},{ }^{3} \mathrm{~J}_{\mathrm{HH}}=10.1 \mathrm{~Hz}, \mathrm{CH}\left(\mathrm{CNCO}_{2} \mathrm{Et}\right)\right)$, 7.50-7.80 (15H, m, $\left.3 \mathrm{xC}_{6} \mathrm{H}_{5}\right) .{ }^{13} \mathrm{C} \mathrm{NMR}(75.5 \mathrm{MHz}): \delta 13.81,14.08$ and $15.02\left(3 \mathrm{~s}, 3 \mathrm{xOCH}_{2} \mathrm{CH}_{3}\right)$, $39.27\left(\mathrm{~d},{ }^{1} J_{\mathrm{PC}}=128.5 \mathrm{~Hz}, \mathrm{P}=\mathrm{C}\right), 39.35\left(\mathrm{~d},{ }^{3} J_{\mathrm{PC}}=3.9 \mathrm{CH}\left(\mathrm{CNCO}_{2} \mathrm{Et}\right)\right), 44.47\left(\mathrm{~d},{ }^{2} J_{\mathrm{PC}}=14.5 \mathrm{~Hz}, \mathrm{P}=\mathrm{C}-\right.$ $\mathrm{CH}), 57.67,61.12$ and $62.14\left(3 \mathrm{~s}, 3 \mathrm{xOCH}_{2} \mathrm{CH}_{3}\right), 117.49(\mathrm{~s}, \mathrm{CN}), 126.66\left(\mathrm{~d},{ }^{1} J_{\mathrm{PC}}=92.4 \mathrm{~Hz}, \mathrm{C}_{\mathrm{ipso}}\right)$, $128.56\left(\mathrm{~d},{ }^{3} J_{\mathrm{PC}}=12.3 \mathrm{~Hz}, \mathrm{C}_{\text {meta }}\right), 132.14\left(\mathrm{~d},{ }^{4} J_{\mathrm{PC}}=2.6 \mathrm{~Hz}, \mathrm{C}_{\mathrm{para}}\right), 134.13\left(\mathrm{~d},{ }^{2} J_{\mathrm{PC}}=9.8 \mathrm{~Hz}, \mathrm{C}_{\text {ortho }}\right)$, $166.39\left(\mathrm{~s}, \mathrm{C}=\mathrm{O}, \mathrm{CO}_{2} \mathrm{Et}\right), 169.15\left(\mathrm{~d},{ }^{2} J_{\mathrm{PC}}=12.8 \mathrm{~Hz}, \mathrm{P}-\mathrm{C}=\mathrm{C}\right), 172.83\left(\mathrm{~d},{ }^{3} J_{\mathrm{PC}}=4.7 \mathrm{~Hz}, \mathrm{C}=\mathrm{O}\right) .{ }^{31} \mathrm{P}$ NMR (121.5 MHz): $\delta 23.19\left(\mathrm{Ph}_{3} \mathrm{P}^{+}-\mathrm{C}\right)$. Minor isomer $(E)-3 \mathrm{e}(33 \%):{ }^{1} \mathrm{H}$ NMR $(300.1 \mathrm{MHz}): \delta$ $0.46\left(3 \mathrm{H}, \mathrm{t},{ }^{3} J_{\mathrm{HH}}=7.0 \mathrm{~Hz}, \mathrm{OCH}_{2} \mathrm{CH}_{3}\right), 1.17-1.24\left(6 \mathrm{H}, \mathrm{m}, 2 \mathrm{xCH}_{3}\right), 3.11\left(1 \mathrm{H}, \mathrm{dd},{ }^{3} J_{\mathrm{HH}}=11.0 \mathrm{~Hz}\right.$, $\left.{ }^{3} J_{\mathrm{PH}}=16.6 \mathrm{~Hz}, \mathrm{P}=\mathrm{C}-\mathrm{CH}\right), 3.71-3.78$ and $4.00-4.20\left(6 \mathrm{H}, 2 \mathrm{~m}, 3 \mathrm{xABX}_{3}\right.$ system, $\left.3 \mathrm{xOCH}_{2} \mathrm{CH}_{3}\right), 4.64$ $\left(1 \mathrm{H}, \mathrm{d},{ }^{3} \mathrm{~J}_{\mathrm{HH}}=11.0 \mathrm{~Hz}, \mathrm{CH}\left(\mathrm{CNCO}_{2} \mathrm{Et}\right)\right), 7.50-7.80\left(15 \mathrm{H}, \mathrm{m}, 3 \mathrm{xC}_{6} \mathrm{H}_{5}\right) .{ }^{13} \mathrm{C} \mathrm{NMR}(75.5 \mathrm{MHz}): \delta$ 13.87, 14.15 and $14.95\left(3 \mathrm{~s}, 3 \mathrm{xOCH}_{2} \mathrm{CH}_{3}\right), 40.44\left(\mathrm{~d},{ }^{1} J_{\mathrm{PC}}=136.9 \mathrm{~Hz}, \mathrm{P}=C\right), 40.80\left(\mathrm{~d},{ }^{3} J_{\mathrm{PC}}=3.9\right.$ $\left.\mathrm{CH}\left(\mathrm{CNCO}_{2} \mathrm{Et}\right)\right), 43.84\left(\mathrm{~d},{ }^{2} \mathrm{~J}_{\mathrm{PC}}=14.3 \mathrm{~Hz}, \mathrm{P}=\mathrm{C}-\mathrm{CH}\right), 58.30,61.19$ and $62.29\left(3 \mathrm{~s}, 3 \mathrm{xOCH}_{2} \mathrm{CH}_{3}\right)$, $117.14(\mathrm{~s}, \mathrm{CN}), 126.03\left(\mathrm{~d},{ }^{1} J_{\mathrm{PC}}=92.4 \mathrm{~Hz}, \mathrm{C}_{\mathrm{ipso}}\right), 128.67\left(\mathrm{~d},{ }^{3} J_{\mathrm{PC}}=12.2 \mathrm{~Hz}, \mathrm{C}_{\text {meta }}\right), 132.14(\mathrm{~d}$, $\left.{ }^{4} J_{\mathrm{PC}}=2.6 \mathrm{~Hz}, \mathrm{C}_{\mathrm{para}}\right), 134.13\left(\mathrm{~d},{ }^{2} J_{\mathrm{PC}}=9.8 \mathrm{~Hz}, \mathrm{C}_{\mathrm{ortho}}\right), 166.13\left(\mathrm{~s}, \mathrm{C}=\mathrm{O}, \mathrm{CO}_{2} \mathrm{Et}\right), 169.86\left(\mathrm{~d},{ }^{2} J_{\mathrm{PC}}=17.7\right.$ $\mathrm{Hz}, \mathrm{P}-\mathrm{C}=C), 173.24\left(\mathrm{~d},{ }^{3} J_{\mathrm{PC}}=5.1 \mathrm{~Hz}, \mathrm{C}=\mathrm{O}\right) .{ }^{31} \mathrm{P}$ NMR $(121.5 \mathrm{MHz}): \delta 23.73\left(\mathrm{Ph}_{3} \mathrm{P}^{+}-\mathrm{C}\right)$.

Di-tert- butyl 2-(ethyl 1-cyanoethanoat-1-yl)-3-(triphenylphosphanylidene)butanedioate (3f). The yield was $0.57 \mathrm{~g}, 95 \%, \mathrm{mp} 144-146^{\circ} \mathrm{C}$, IR $(\mathrm{KBr})\left(v_{\max }, \mathrm{cm}^{-1}\right): 2270(\mathrm{CN}), 1727,1730$ and $1616(\mathrm{C}=\mathrm{O})$. MS (m/z, \%): $601\left(\mathrm{M}^{+}, 10\right), 500(22), 489(20), 262(23), 183(59), 108(18), 77$ (9), 57 (100). Anal. Calcd for $\mathrm{C}_{35} \mathrm{H}_{40} \mathrm{NO}_{6} \mathrm{P}$ (601.67): C, 69.87; H, 6.70; N, 2.33. Found: $\mathrm{C}, 69.75$; $\mathrm{H}, 6.708$; $\mathrm{N}, 2.41 \%$. Major isomer (Z)- 3f $(70 \%):{ }^{1} \mathrm{H}$ NMR $(300.1 \mathrm{MHz}), \delta 0.95$ and $1.43(18 \mathrm{H}$, $2 \mathrm{~s}, 2 \mathrm{x} \mathrm{CMe}), 1.19\left(3 \mathrm{H}, \mathrm{t},{ }^{3} J_{\mathrm{HH}}=7.1 \mathrm{~Hz}, \mathrm{OCH}_{2} \mathrm{CH}_{3}\right), 2.84\left(1 \mathrm{H}, \mathrm{dd},{ }^{3} J_{\mathrm{HH}}=10.9 \mathrm{~Hz},{ }^{3} J_{\mathrm{PH}}=18.7 \mathrm{~Hz}\right.$, $\mathrm{P}=\mathrm{C}-\mathrm{CH}), 4.10\left(2 \mathrm{H}, \mathrm{m}, \mathrm{ABX}_{3}\right.$ system, $\left.\mathrm{OCH}_{2} \mathrm{CH}_{3}\right), 5.01\left(1 \mathrm{H}, \mathrm{d},{ }^{3} \mathrm{~J}_{\mathrm{HH}}=10.9 \mathrm{~Hz}, \mathrm{CH}\left(\mathrm{CNCO}_{2} \mathrm{Et}\right)\right)$,

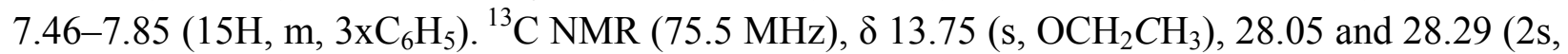
2x $\left.\mathrm{CMe}_{3}\right), 39.07\left(\mathrm{~d},{ }^{1} J_{\mathrm{PC}}=129.1 \mathrm{~Hz}, \mathrm{P}=\mathrm{C}\right), 39.41\left(\mathrm{~d},{ }^{3} \mathrm{JC}_{\mathrm{PC}}=3.9 \mathrm{~Hz}, \mathrm{CH}\left(\mathrm{CNCO}_{2} \mathrm{Et}\right)\right), 45.37$ (d, $\left.{ }^{2} J_{\mathrm{PC}}=14.8 \mathrm{~Hz}, \mathrm{P}=\mathrm{C}-\mathrm{CH}\right), 61.85\left(\mathrm{~s}, \mathrm{OCH}_{2} \mathrm{CH}_{3}\right), 77.38$ and $80.92(2 \mathrm{~s}, 2 \mathrm{x} \mathrm{OCMe}), 117.95(\mathrm{~s}, \mathrm{CN})$, $126.49\left(\mathrm{~d},{ }^{1} J_{\mathrm{PC}}=92.4 \mathrm{~Hz}, \mathrm{C}_{\mathrm{ipso}}\right), 128.36\left(\mathrm{~d},{ }^{3} J_{\mathrm{PC}}=11.9 \mathrm{~Hz}, \mathrm{C}_{\text {meta }}\right), 131.96\left(\mathrm{~d},{ }^{4} J_{\mathrm{PC}}=2.4 \mathrm{~Hz}, \mathrm{C}_{\mathrm{para}}\right)$, $134.24\left(\mathrm{~d},{ }^{2} J_{\mathrm{PC}}=9.5 \mathrm{~Hz}, \mathrm{C}_{\text {ortho }}\right), 166.40\left(\mathrm{C}=\mathrm{O}, \mathrm{CO}_{2} \mathrm{Et}\right), 168.57\left(\mathrm{~d},{ }^{2} J_{\mathrm{PC}}=12.3 \mathrm{~Hz}, \mathrm{P}-\mathrm{C}=C\right), 171.96$ 
$\left(\mathrm{d},{ }^{3} J_{\mathrm{PC}}=5.0 \mathrm{~Hz}, \mathrm{C}=\mathrm{O}\right) .{ }^{31} \mathrm{P}$ NMR $(121.5 \mathrm{MHz}): \delta 22.56\left(\mathrm{Ph}_{3} \mathrm{P}^{+}-\mathrm{C}\right)$. Minor isomer $(E)-3 \mathbf{f}(30 \%)$ :

${ }^{1} \mathrm{H}$ NMR $(300.1 \mathrm{MHz}), \delta 1.41$ and $1.45\left(18 \mathrm{H}, 2 \mathrm{~s}, 2 \mathrm{xCMe}_{3}\right), 1.21\left(3 \mathrm{H}, \mathrm{t},{ }^{3} \mathrm{~J}_{\mathrm{HH}}=7.1 \mathrm{~Hz}\right.$, $\left.\mathrm{OCH}_{2} \mathrm{CH}_{3}\right), 2.91\left(1 \mathrm{H}, \mathrm{dd},{ }^{3} J_{\mathrm{HH}}=10.8 \mathrm{~Hz},{ }^{3} J_{\mathrm{PH}}=17.1 \mathrm{~Hz}, \mathrm{P}=\mathrm{C}-\mathrm{CH}\right), 4.10\left(2 \mathrm{H}, \mathrm{m}, \mathrm{ABX}_{3}\right.$ system, $\left.\mathrm{OCH}_{2} \mathrm{CH}_{3}\right), 4.71\left(1 \mathrm{H}, \mathrm{d},{ }^{3} \mathrm{~J}_{\mathrm{HH}}=10.8 \mathrm{~Hz}, \mathrm{CH}\left(\mathrm{CNCO}_{2} \mathrm{Et}\right)\right), 7.51-7.81\left(15 \mathrm{xH}_{\text {arom }}, \mathrm{m}, 3 \mathrm{x} \mathrm{C}_{6} \mathrm{H}_{5}\right) \cdot{ }^{13} \mathrm{C}$ $\operatorname{NMR}(75.5 \mathrm{MHz}), \delta 13.75\left(\mathrm{~s}, \mathrm{OCH}_{2} \mathrm{CH}_{3}\right), 28.17$ and $28.84(2 \mathrm{~s}, 2 \mathrm{x} \mathrm{CMe}), 40.48\left(\mathrm{~d},{ }^{3} \mathrm{~J}_{\mathrm{PC}}=3.9 \mathrm{~Hz}\right.$, $\left.\mathrm{CH}\left(\mathrm{CNCO}_{2} \mathrm{Et}\right)\right), 41.01\left(\mathrm{~d},{ }^{1} J_{\mathrm{PC}}=136.3 \mathrm{~Hz}, \mathrm{P}=\mathrm{C}\right), 44.28\left(\mathrm{~d},{ }^{2} J_{\mathrm{PC}}=14.7 \mathrm{~Hz}, \mathrm{P}=\mathrm{C}-\mathrm{CH}\right), 62.08(\mathrm{~s}$, $\left.\mathrm{OCH}_{2}\right), 77.63$ and $\left.80.86(2 \mathrm{~s}, 2 \mathrm{x} \mathrm{OCMe})_{3}\right), 117.37(\mathrm{~s}, \mathrm{CN}), 127.16\left(\mathrm{~d},{ }^{1} J_{\mathrm{PC}}=92.5 \mathrm{~Hz}, \mathrm{C}_{\mathrm{ipso}}\right), 128.52$ $\left(\mathrm{d},{ }^{3} J_{\mathrm{PC}}=11.5 \mathrm{~Hz}, \mathrm{C}_{\text {meta }}\right), 131.96\left(\mathrm{~d},{ }^{4} J_{\mathrm{PC}}=2.4 \mathrm{~Hz}, \mathrm{C}_{\mathrm{para}}\right), 134.11\left(\mathrm{~d},{ }^{2} J_{\mathrm{PC}}=9.4 \mathrm{~Hz}, \mathrm{C}_{\text {ortho }}\right), 166.08$ $(\mathrm{C}=\mathrm{O}), 170.07\left(\mathrm{~d},{ }^{2} J_{\mathrm{PC}}=17.9 \mathrm{~Hz}, \mathrm{P}-\mathrm{C}=\mathrm{C}\right), 171.79\left(\mathrm{~d},{ }^{3} J_{\mathrm{PC}}=5.2 \mathrm{~Hz}, \mathrm{C}=\mathrm{O}\right) .{ }^{31} \mathrm{P}$ NMR $(121.5 \mathrm{MHz})$ : $\delta 22.40\left(\mathrm{Ph}_{3} \mathrm{P}^{+}-\mathrm{C}\right)$.

\section{Acknowledgements}

The authors gratefully acknowledge financial support from the Research Council of the University of Sistan \& Balouchestan.

\section{References}

1. (a) Engel, R. Synthesis of Carbon-Phosphorus Bonds; CRC: Boca Raton, FL, 1988. (b) Maryano, B. E.; Reitz, A. B. Chem. Rev. 1989, 89, 863. (c) Bestmann, H. J.; Vostrowsky, O. Top. Curr. Chem. 1983, 109, 86. (d) Grayson, M.; Griffith, E. J. Topics in Phosphorus Chemistry; Interscience: New York, 1972; Vol. 7. (e) Yavari, I.; Maghsoodlou, M. T. Tetrahedron Lett. 1998, 39, 4579. (f) Yavari, I.; Islami, M. R.; Bijanzadeh, H. R. Tetrahedron Lett. 1999, 55, 5547.

2. (a) Ware, E. Chem. Rev. 1950, 403. (b) Spinks, A.; Waring, W. S. Prog. Med. Chem. 1963, 3, 313. (c) Mizuno, T.; Kino, T.; Ito, T.; Miato, T. Synth. Commun. 2000, 30, 1675. (d) Engel, R. Chem. Rev. 1977, 77, 349. (e) Wittig, G. Science 1980, 210, 600.

3. Kolodiazhnyi, O. I. Russ. Chem. Rev. 1997, 66, 225.

4. Pietrusiewiz, K. M.; Zablocka, M. Chem. Rev. 1994, 94, 1375.

5. (a) Yavari, I.; Nasiri, F.; Djahaniani, H. Phosphorus, Sulfur, and Silicon, 2003, 178, 2627. (b) Yavari, I.; Bayat, M. Tetrahedron Lett. 2003, 59, 2001.

6. Cadogan, J. I. G. Organophosphorus Reagents in Organic Synthesis, Academic Press: New York, 1979.

7. Quin, L. D. A Guide to Organophosphorus Chemistry, Wiley-Interscience, New York, 2000.

8. Hudson, H. R. The Chemistry of Organophosphorus Compounds, Primary, Secondary, and Tertiary Phosphates and Heterocyclic Organophosphorus (III) Compounds, Hartley, F. R., Ed.; Wiley: New York, 1990; Vol. 1, pp 382-472.

9. Nicolaou, K. C.; Harter, M. W.; Gunzner, J. L.; Nadin, A. Liebigs Annalen 1997, 7, 1283. 
10. Johnson, A. W. Ylide Chemistry, Academic Press, London, 1966.

11. Corbridge, D. E. C. Phosphorus: an Outline of Chemistry, Biochemistry and Uses, $5^{\text {th }}$ Edn.; Elsevier: Amsterdam, 1995.

12. Maghsoodlou, M. T.; Hazeri, N.; Habibi Khorassani, S. M.; Nassiri, M.; Marandi, G.; Afshari, G.; Niroumand, U. J. Sulfur Chem. 2005, 26, 261.

13. Islami, M. R.; Mollazehi, F.; Badiei, A.; Sheibani, H. ARKIVOC 2005, (xv), 25.

14. Maghsoodlou, M. T.; Hazeri, N.; Habibi Khorassani, S. M.; Afshari, G.; Nassiri, M.; J. Chem. Res. (S) 2005, 727.

15. Hazeri, N.; Habibi Khorassani, S. M.; Maghsoodlou, M. T.; Marandi, G.; Nassiri, M.; Gulam-Shahzadeh, A. J. Chem. Res. (S). 2006, 215.

16. Maghsoodlou, M. T.; Saghatforoush, L.; Aminkhani, A.; Marandi, G.; Kabiri, R. J. Sulfur Chem. 2006, 27, 583.

17. Maghsoodlou, M. T.; Hazeri, N.; Habibi Khorassani, S. M.; Kakaei, R.; Nassiri, M.; Marandi, G.; Moeeni, Z.; Niromand, U.; Z. Eskandari-Torbaghan, Phosphorus, Sulfur, and Silicon 2006, 181, 865.

18. Kalantari, M.; Islami, M. R.; Hassani, Z.; Saidi, K. ARKIVOC 2006, (x), 55.

19. Hassani, Z.; Islami, M. R.; Sheibani, H.; Kalantari, M.; Saidi, K. ARKIVOC 2006, (i), 89.

20. Maghsoodlou, M. T.; Hazeri, N.; Habibi Khorassani, S. M.; Saghatforoush, L.; Rofouei, M. K.; Rezaie, M. ARKIVOC 2006, (xiii), 117.

21. Maghsoodlou, M. T.; Habibi Khorassani, S. M.; Hazeri, N.; Nassiri, M. Phosphorus, Sulfur, and Silicon 2006, 181, 1363.

22. Esmaili, A. A.; Ghereghloo, M.; Islami, M. R.; Bijanzadeh, H. R. Tetrahedron 2003, 59, 4785.

23. Bestmann, H. J.; Joachim, G.; Lengyel, T.; Oth, J. F.; Merenyi, R.; Weitkamp, H. Tetrahedron Lett. 1966, 3355.

24. Islami, M. R.; Yavari, I.; Tikdari, A. M.; Ebrahimi, L.; Razee, S.; Bijanzadeh, H. R. Russ. Chem. Bull. 2002, 51, 2244. 